Classification

Physics Abstracts

$07.80-61.16 \mathrm{~B}-61.46$

\title{
Letter
}

\section{HREM characterization of graphitic nanotubes}

\author{
Daniel Ugarte
}

Laboratorio Nacional de Luz Síncrotron (CNPq/MCT), Caixa Postal 6192, 13081-970 Campinas SP, Brazil and Institut de Physique Expérimentale, Ecole Polytechnique Fédérale de Lausanne, 1015 Lausanne, Switzerland

(Received 21 January 1994; accepted 25 January 1994)

\begin{abstract}
We use high resolution electron microscopy to study the structure of carbon nanotubes. Although the great scientific and technologic interest generated by these nanometric structures, their growth mechanism remains not completely understood. We study the atomic structure of the tubes and in particular we concentrate on some frequent defective configurations in order to get some insight into the formation process. A very interesting case is constituted by nanotubes showing irregular (0.34$0.6 \mathrm{~nm}$ ) interlayer spacing, this fact would allow the doping of the tubes, and probably the optimization of their electric properties.
\end{abstract}

\section{Introduction.}

Fullerenes [1,2] have opened a new dimension on the way of looking at graphitic networks. At present, it is well accepted that carbon atoms with a $\mathrm{sp}^{2}$ like hybridization may form curved surfaces with nanometric diameter, and moreover that the traditional hexagonal graphitic network may incorporate non-six-member rings (pentagons and/or heptagons). High Resolution Electron Microscopy (HREM) has been revealed as an invaluable tool for the study of fullerene-related materials, such as nanotubes [3], or polyhedral graphitic particles [4-6].

In particular, graphitic nanotubes have generated a great deal of interest both from the basic and applied point of view. These tubules are constituted by graphene sheets rolled cylindrically and arranged coaxially with an interlayer distance of $0.34 \mathrm{~nm}$. Their good crystallization suggests their utilization as the strongest fibers ever fabricated. In particular, nanotubes display a very interesting correlation between structural parameters and electronic properties: they may be insulators, semiconductors or metallic as a function of radii and helicity of the hexagon arrangement [7-9]. Originally, Iijima discovered their formation on the surface of the graphite electrodes used in an arc discharge [3], later this procedure was optimized to produce macroscopic quantity 
of nanotubes [10]. The last twist in the world of carbon tubules is the generation of single-shell tubes by using iron- or cobalt-containing electrodes in the arc [11,12].

The arc-discharge experiment constitutes an easy and cheap method to synthesize nanotubes, nevertheless the control of the tube growth is very intricate in the chaotic plasma generated by the electric arc. Additionally, electron irradiation experiments seem to indicate that spherical structures would be more stable than the tubular ones [13]. Therefore, the high efficiency of nanotube generation in the modified Krätschmer-Huffman electric arc raises several intriguing questions. Smalley has emphazised the important role of the electric field in the stabilization of the tubular geometry during its growth [14]. Recently, the formation of cylindrical graphitic particles (short nanotubes) as a byproduct of thermal annealing of fullerene black have also been reported [15].

In this work, we study the structure of nanotubes by high resolution electron microscopy. We analyze some particular frequent defective configuration, and try to gather some information about the growth conditions in the electric arc. The understanding of the different parameters involved would allow the development of a novel nanometric carbon engineering.

\section{Experiments.}

The graphitic tubes used in this study were obtained from an arc-discharge apparatus using graphite electrodes of $6 \mathrm{~mm}$ in diameter, sharpened to $4 \mathrm{~mm}$ at the ends, which are heated by an alternate current $(15 \mathrm{~V}, 150-200 \mathrm{~A})$ in a $300 \mathrm{mbar} \mathrm{He}$ or Ar atmosphere. We scrapped the surface of contact between the electrodes, and the powder generated was dry deposited on holey carbon grids.

The observation were carried out using a Philips EM 430 ST with a resolution of $0.2 \mathrm{~nm}$, allowing the imaging of the (002) lattice planes of graphite $\left(d_{002}=0.34 \mathrm{~nm}\right)$. The vacuum in the specimen chamber is $\approx 10^{-7}$ torr.

In figure 1, we present high resolution images of typical tubular structures formed in an arcdischarge experiment. Their diameter is in the 3-40 $\mathrm{nm}$ range, which means 2-50 coaxial tubes. The tubular structure is verified by the appearance of the same number of lattice fringes in both sides of a tubule in the HREM image. The inner tube of the needle may have diameter as small as $\mathrm{C}_{60}$. Although the wide size distribution of tubes sizes, we have noticed that tubes showing very small ( $\leq 1 \mathrm{~nm}$ ) inner tubes were composed of more than 10 layers, while tubes formed by few layers (2-3) always presented larger inner space 3-4 nm (see Fig. 1a); these two characteristics do not exclude that tubes with large inner space (3-4 $\mathrm{nm}$ in diameter) may also present thick walls (composed of many graphitic layers). No single walled tube was observed with the use of pure carbon electrodes.

The graphitic needle tips are closed by quasi-hemispherical or polyhedral graphitic domes. The morphology of the cap displays the distribution of pentagons on the surface of the tip [16]. A current configuration appearing in tubular structures, is constituted by the closure of some inner tubes by a hemispherical cap and the formation a second cap in opposite direction ("bamboo-like defect" [17]), dividing the inner hollow space in several compartments (Fig. 1b). It is noticeable that in most cases both caps present equal number of layers, but in rare cases slight differences of 1-2 layers may occur.

In most of the tubes, the register of the graphitic layers is very good, even in extremely curved regions containing positive or negative curvature (pentagons or heptagons). Detailed examination of the samples shows that in rare cases tubular structures may present a perturbed register between layers (Figs. 4 and 5). Figure 5 shows the tip of tubule formed by 19 coaxial tubes. The upper part of the picture displays a very good crystallization, but the lower side, presenting also 19 planes, shows an irregular interlayer distance, varying between $0.34-0.6 \mathrm{~nm}$. 


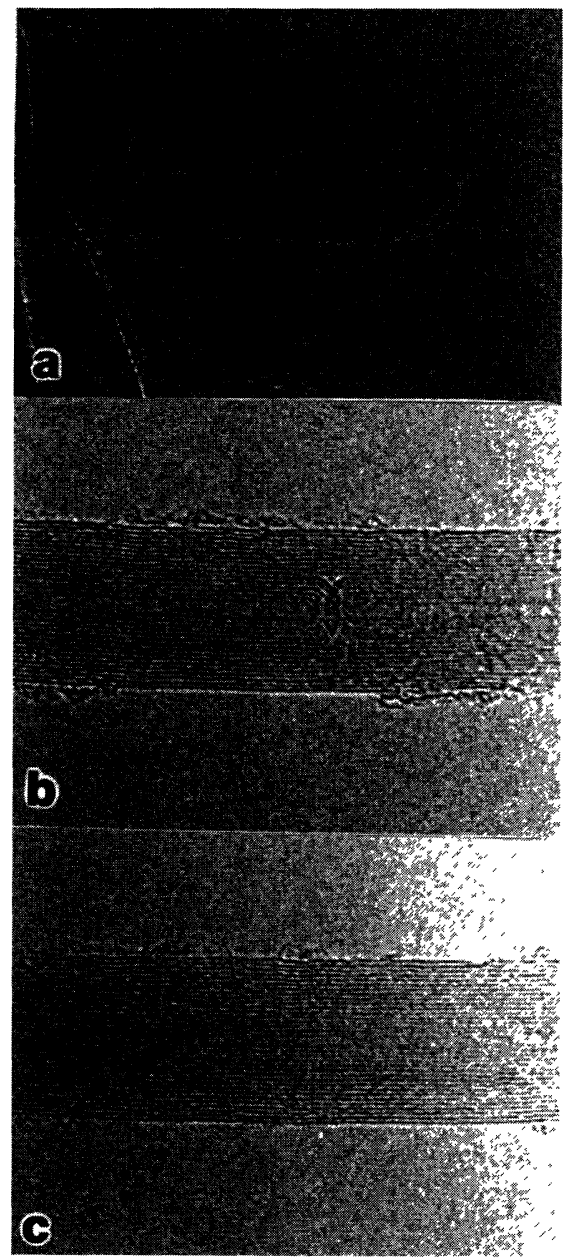

Fig. 1. - High resolution electron micrographs of buckytubes. a) Three layer tubule (inner diameter $\approx 3$ $\mathrm{nm}$ ); b) 16-shelled tube (inner diameter $\approx 0.12 \mathrm{~nm}$ ) presenting a bamboo defect (closure of the 6 innermost tubes), note the superposition of the two inner caps, that are symmetrical in the image plane, but must be asymmetrical in the observation direction; c) 17 layer tube, with a very small central shell $(\approx 0.7 \mathrm{~nm})$ central tube (interlayer distance is $0.34 \mathrm{~nm}$ ).

\section{Discussion.}

The interpretation of high resolution electron micrographs usually requires a comparison with numerical simulations. In the case of the particles studied here, the understanding of the observed contrasts may be easily related to the graphitic structure (black lines correspond to atomic positions in all the figures). The remarkable fact is that even for tubules with small diameters ( $\leq$ $1 \mathrm{~nm}$ ), a good contrast is observable. The signal is generated by sectors of the tubular structures parallel to the electron beam containing only 2-3 carbons atoms. Considering that the elastic cross section for such a low atomic number element is very low $\left(\sigma_{\text {elast }} \propto Z^{4 / 3}\right)$, the imaging of such a system is a very good example of the actual power of high resolution electron microscopes.

Theoretical results have shown that a monolayer tubule is more stable that the flat sheet re- 
sulting from unrolling the tubule up to a certain minimum radius, where the energy stored in the bending of the strip is smaller than the energy gained by the dangling bond elimination $[18,19]$. The amount of bending energy represents an energy barrier for the closure to occur. This barrier can be overcome by thermal fluctuations [20], the higher the temperature, the smaller the radius of the formed graphitic tube. Similar conclusions may be obtained from the analysis of carbon onions generated by thermal annealing of carbon soot, that have clearly shown that the inner shell diameter decreases as the temperature of the treatment increases [15].

We may transpose these observations to the electric arc: low temperature regions of the electrode would yield tubes with larger diameters. It is accepted that the tubes grow thicker by adding graphitic islands simultaneously at different points on the surface of the tube, that coalesce later to add a new cylindrical layer [17]. Then, considering that colder regions would present lower carbon vapor density, it would result thinner walled tubes with relatively large inner empty space (3-4 nm diameter). These speculations agree with the observations (see Fig. 1). The structural parameters of the nanotube (inner diameter and number of shells) may possibly be correlated to the temperature of the surface of the electrode.

The electric-arc is usually a transient process where the regions of the electrode surface generating the arc changes permanently all over the surface of contact. The arc-discharge experiment generates a range of temperatures on the electrode surface, thus yielding a large size distribution of tubes. Monodispersed and controlled tubule generation should be produced within an equipment guaranteeing a more stable temperature regime. However, we must notice this is very difficult to implement in practice, due to the high temperature necessary to produce carbon vapor $(\approx 4000 \mathrm{~K})$.

The Ebbesen-Ajayan method to produce nanotubes [10] yields a package of parallel tubes arranged in parallel bundles [21]. This arrangement, as well as the formation of structures with such a high length/diameter ratio $(\approx 100)$, is suggested to be due to the high electric field produced between the plasma and the surface of the graphite electrode $\left(E \approx 10^{6}-10^{8} \mathrm{~V} \mathrm{~cm}^{-1}\right)[6,14]$.

As for the growth mechanism of buckytubes, recent open tube observations [17] have given weight to the open end model, where the tube grows longer by adding atoms to an open tip. Smalley [14] has stressed the important role of the high electric field between the electrodes, the value of the field is of the same magnitude as in a chemical bond, therefore it would stabilize the open tube tip during growth. Another growth model based on the adding of carbon atoms to a closed tip seems less plausible [22], because it is rather difficult to explain the growth of the inner layers. Bamboo-like defects, as shown in figure $1 \mathrm{~b}$, also imply that the growth may be developed from the open tip in backwards direction, generating consequently the second spherical cap. In most cases, the number of shells at both sides of the bamboo-defect is equal, indicating that somewhat an ensemble of parameters determines the inner and outermost radii of the tubular structure, as for example the temperature or pressure of carbon vapor in the plasma, as discussed previously.

Most of the theoretical predictions for buckytubes have dealed with a well defined cylindrical shape. It has been proposed that in multilayer tubes, the energy barriers for sliding or rotation are very low, hence at room temperature coaxial tubules may be rotating [23]. Nevertheless, cross section imaging of buckytubes show that they may not be perfectly circular [24-25]. Recently the radial deformation of adjacent tubes have been observed [26], the driving force for this radial deformation is the optimization of the Van der Waals attraction forces between the external graphitic layers of the tubes.

The importance of Van der Waals energy in the energetics of multilayer fullerene-related structures must be emphasized. The VdW interaction between layers $(1.5-2.0 \mathrm{kcal} / \mathrm{mol}$ per atom for bulk graphite) is responsible for the robustness of nanometric multishelled onion-like particles $[13,27]$. Similar properties may be observed in the closure of some inner layers in tubes. The 


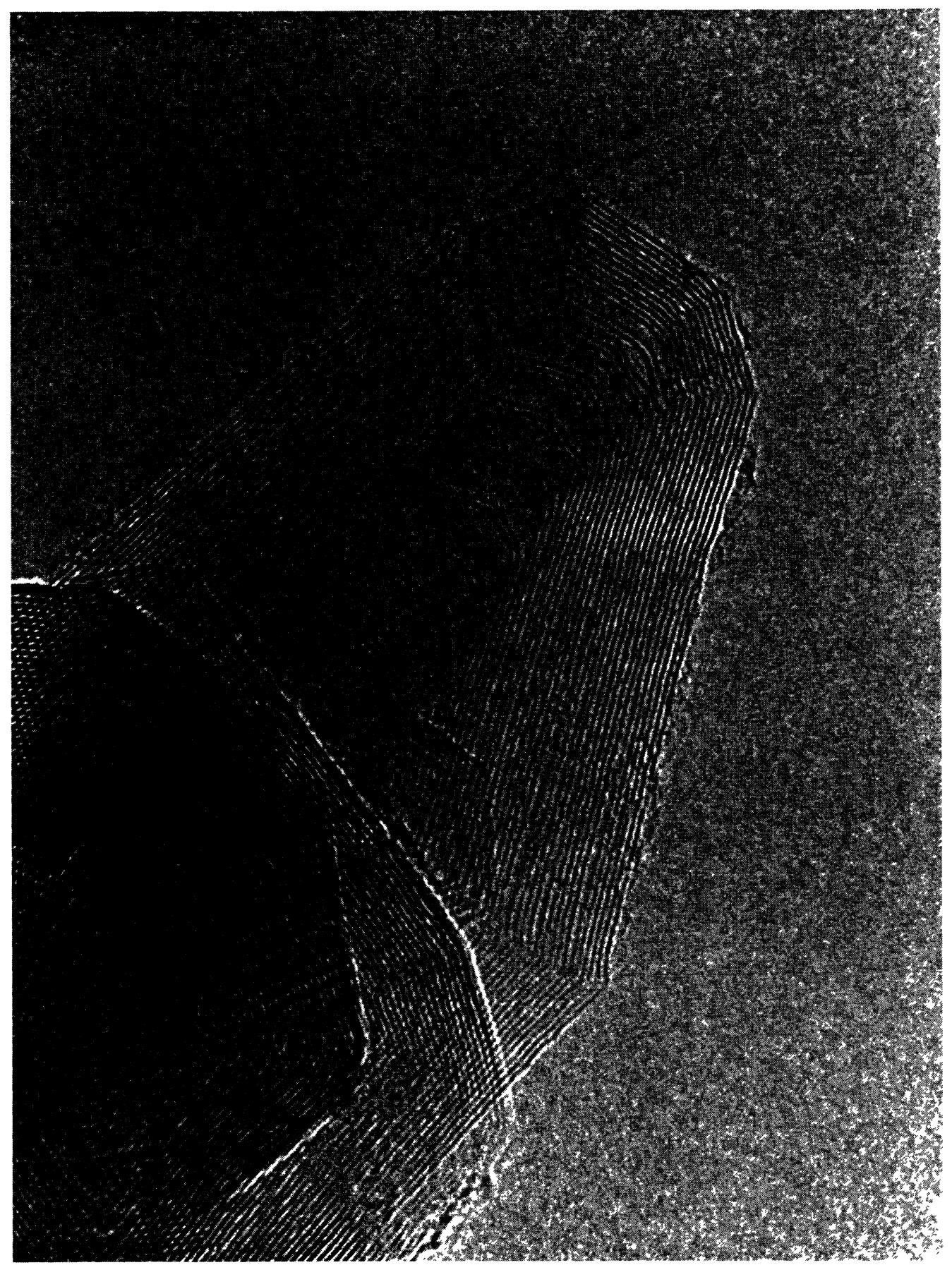

Fig. 2. - Conical tip of a nanotube displaying progressive partial closure of the carbon layers (interlayer distance is $0.34 \mathrm{~nm}$ ). Note that most of the closures are formed by a couple of graphene sheets.

closure is made usually by two or more layers, indicating the rather strong instability of a single curved graphitic dome. This fact is clearly exemplified in figure 2 , where we show the tip of a 
buckytube displaying several partial closures, each one made by a two layers cap. In rare cases, closure may be produced by a single graphite sheet (Fig. 3).

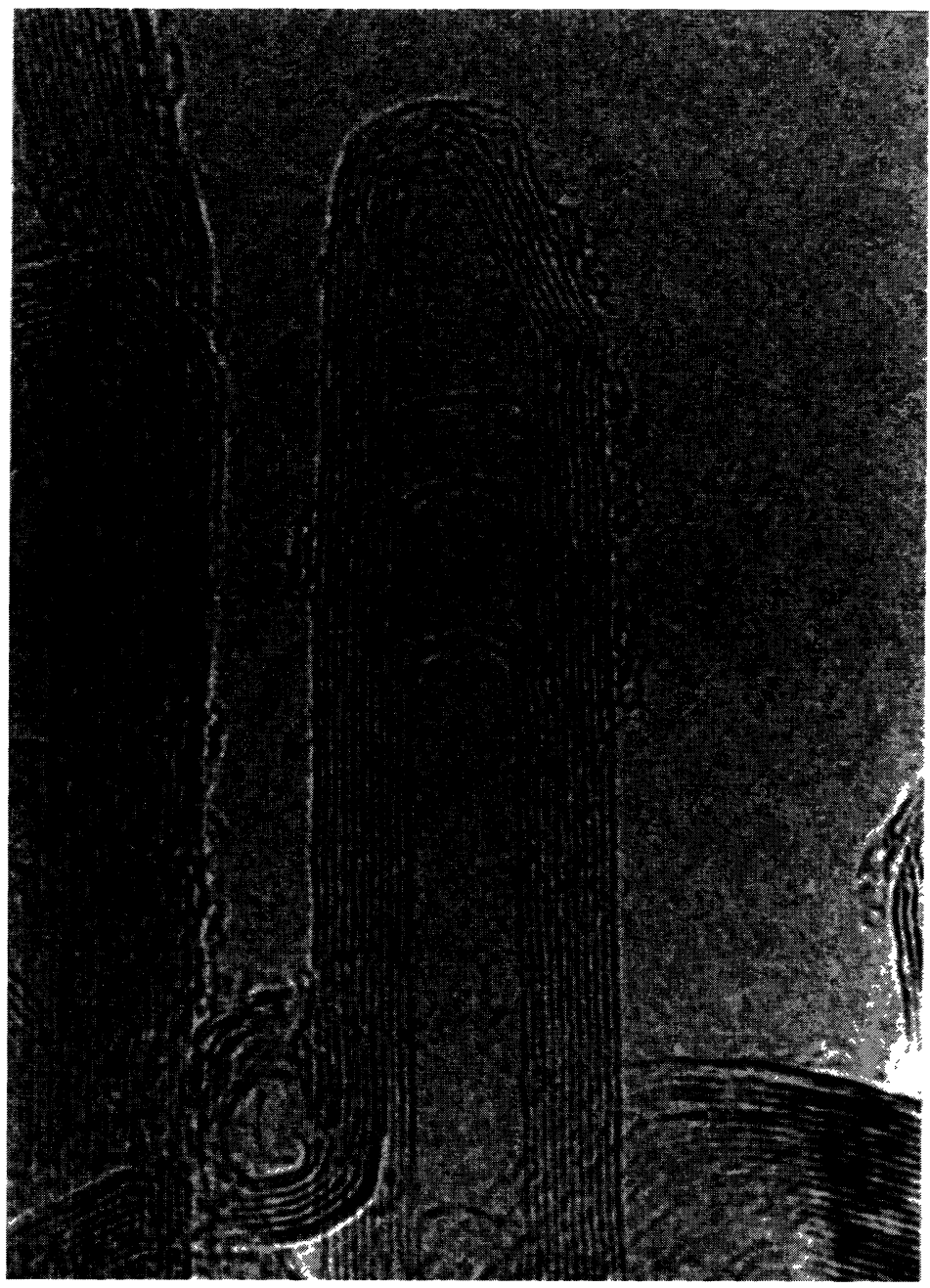

Fig. 3. - Carbon tubule tip. Single shell caps formed in the inner region, this configuration is much less frequent than multilayer caps (interlayer distance is $0.34 \mathrm{~nm}$ ).

Doping curved and closed concentric graphitic structures is not easy compared to planar graphite. The dopant induces an increase of the interlayer spacing, that implies the increase of the diameters of the tubes, therefore producing the breakdown of the structure. Here, we have observed the occurrence of variations in the interlayer spacing (0.34-0.6 $\AA$, Figs. 4 and 5). This larger interlayer distance associated with the possibility of opening the tube tips by oxidation [28,29], may be used to introduce interstitial atoms in the tubes in order to modify their electronic properties. 


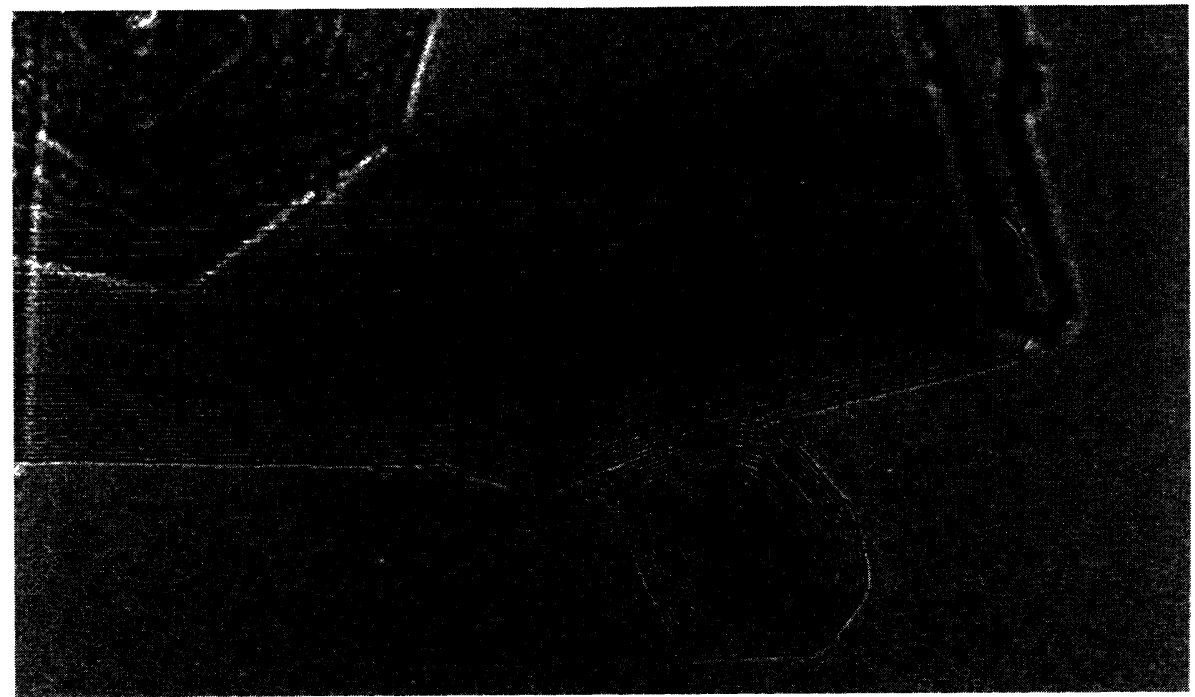

Fig. 4. - Nanotube showing irregular spacing of the coaxial layers (interlayer distance is $0.34 \mathrm{~nm}$ in the lower side of the tube).

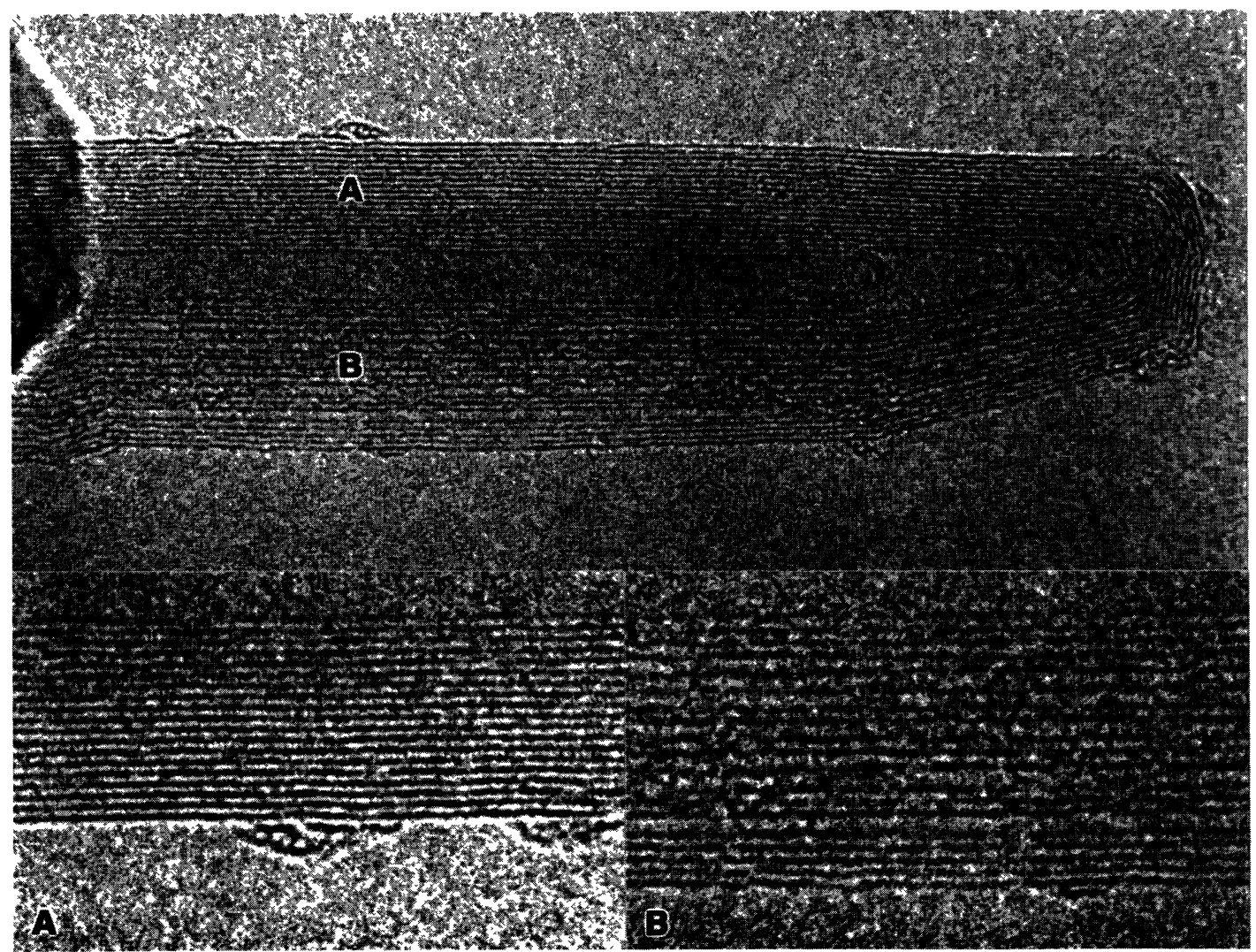

Fig. 5. - Defective tubule displaying irregular register of the graphitic layers. In the lower part of the figure we may see details of the tube wall (marked A and B) for an easy eye comparison of the layers spacing. 
Recently the generation of single-shell tubes was reported by using metal-carbon composed electrodes $[11,12]$. In this case, the formation mechanism seems different, and mostly similar to carbon fiber production by thermal decomposition of hydrocarbons on a catalytic particle [30]. The size of the metallic particle defines the size of the graphitic tube.

In summary, HREM has been used to study the detailed atomic structure of carbon nanotubes. On the basis of the observations, we speculate that there exists a certain relation between the diameter of the inner tube and the number of layers constituting the tube. We associate this correlation to the temperature of the region where the tube was formed. Tubes presenting irregular spacing between layers may be used for intercalation compounds, generating buckytubes with optimized electric properties.

\section{Acknowledgements.}

We wish to acknowledge the contributions of W. de Heer and D. Reinhard in offering valuable suggestions and stimulating discussions during the course of this work. We thank R. Monot and A. Chatelain for several useful remarks. We are indebted to the Brazilian Council for Scientific and Technologic Research (CNPq) and the Swiss National Science Foundation for financial support.

\section{References}

[1] KRoto H.W., HeATH J.R., O’BRIEN S.C., CURL R.F., SMALley R.E., Nature 318 (1985) 162.

[2] KRÄTSCHMER W., LAMB L.D., FORISTOPOULOS K., HuFFMAN D.R., Nature 347 (1990) 354.

[3] IIJIMA S., Nature 354 (1991) 56.

[4] IIJIMA S., J. Cryst. Growth 50 (1980) 675.

[5] UgarTe D., Chem. Phys. Lett. 198 (1992) 596.

[6] Saito Y., Yoshikawa T., Inagaki M., Tomita M., Hayashi T., Chem. Phys. Lett. 204 (1993) 277.

[7] MinTMIRE J.W., DunLAP B.I., White C.T., Phys. Rev. Lett. 68 (1992) 631.

[8] Hamada N., Sawada S., OshiYama A., Phys. Rev. Lett. 68 (1992) 1579.

[9] Tanaka K., Okahara K., OKada M., Yamabe T., Chem. Phys. Lett. 191 (1992) 469.

[10] EBBESEN T.W., AJAYAN P.M., Nature 358 (1992) 220.

[11] IIJIMA S., ICHIHASHI T., Nature 363 (1993) 603.

[12] Bethune D.S., Kiang C.H., de VRIES M.S., Gorman G., SAVoy R., VAZQueZ J., Beyers R., Nature 363 (1993) 605.

[13] UGARTE D., Nature 359 (1992) 707.

[14] SMALley R.E., Mater. Sci. Eng. B19 (1993) 1.

[15] de HeEr W.A., Ugarte D., Chem. Phys. Lett. 207 (1993) 480.

[16] AJAYAN P.M., ICHIHASHI T., IIJima S., Chem. Phys. Lett. 202 (1993) 384.

[17] IIJIMA S., AJAYAN P.M., ICHIHASHI T., Phys. Rev. Lett. 69 (1992) 3100.

[18] Sawada S., Hamada N., Sol. St. Com. 83 (1992) 917.

[19] Lucas A.A., Lambin P.H., SMalley R.E., J. Phys. Chem. Solids 54 (1993) 587.

[20] RoberTSOn D.H., BRENNER D.W., White C.T., J. Phys. Chem. 96 (1992) 6133.

[21] WANG X.K., Lin X.W., Dravid V.P., KeTterson J.B., ChANG R.P.H., Appl. Phys. Lett. 62 (1993) 1881.

[22] Endo M., Kroto H.W., J. Phys. Chem. 96 (1992) 6941.

[23] Charlier J.-C., Michenaud J.-P., Phys. Rev. Lett. 70 (1993) 1858.

[24] HARRIS P.J.F., GreEN M.L.H., TSANG S.C., J. Chem. Soc. Faraday Trans. 89 (1993) 1189.

[25] Dravid V.P., LIN X., Wang X.Y., WANG X.K., Yee A., KetTerson J.B., Chang R.P.H., Science 259 (1993) 1601.

[26] RuOfF R.S., Tersoff J., Lorents D.C., Subramoney S., Chan B., Nature 364 (1993) 514.

[27] UgARTE D., Europhys. Lett. 22 (1993) 45.

[28] AJAYAN P.M., EBbESEn T.W., ICHIHASHI T., IIJIMA S., TANIGAKI K., HIURA H., Nature 362 (1993) 522.

[29] TSANG S.C., HARRIS P.J.F., GreEN M.L.H., Nature 362 (1993) 520.

[30] TibBets G.G., J. Cryst. Growth 66 (1984) 632. 